\title{
Der Arbeitsmarkt in der Großen Rezession - Bewährte Strategien in neuen Formen
}

\author{
Alexander Herzog-Stein \\ Hartmut Seifert
}

In der Wirtschaftkrise hat sich gezeigt, dass der deutsche Arbeitsmarkt weitaus flexibler ist als häufig unterstellt wird. Ohne die vielfältigen, in den Jahren vor der Krise von den Tarifvertragsparteien geschaffenen Möglichkeiten, die Arbeitszeit flexibel einzusetzen, hätte der heftige Konjunkturabschwung von 2008/09 die Arbeitslosigkeit steil ansteigen lassen. Der Beitrag untersucht, wie sich die neuen Formen flexibler Arbeitszeitgestaltung von früheren unterscheiden und welchen Beitrag sie zur Beschäftigungssicherung leisten.

\section{Einleitung}

Angesichts der robusten Arbeitsmarktsituation während der schärfsten Nachkriegskrise, der sogenannten Großen Rezession, ${ }^{1}$ ist vom deutschen Beschäftigungswunder die Rede (Krugman 2009; Möller 2010). Das vermeintliche Wunder ist rasch aufgeklärt. Die vor allem im internationalen Vergleich stabile Entwicklung von Beschäftigung und Arbeitslosigkeit lässt sich auf den verstärkten Einsatz intern-numerischer Instrumente sowie das Horten von Arbeitskräften zurückführen (Walwei 2010; Herzog-Stein/Seifert 2010; OECD 2010). Im Prinzip sind diese Strategien, mit denen Betriebe auf Nachfrageeinbrüche reagieren, nicht neu. Anpassungen des Arbeitseinsatzes durch Variation der Arbeitszeit haben in konjunkturellen Abschwungphasen Tradition. Bereits in der Krise 1973/75 spielten Arbeitszeit verkürzende Instrumente eine bedeutsame Rolle bei der Beschäftigungssicherung. Seitdem hat sich jedoch der rechtlich-institutionelle Rahmen des Arbeitsmarktes verändert. Gesetzgeber und Tarifvertragsparteien erweiterten den Handlungsspielraum für betriebliche Flexibilität. Bestehende Instrumente, den Arbeitseinsatz an eine volatile Nachfrage anpassen zu können, wurden flexibler ausgerichtet und neue eingeführt, sodass insgesamt mehr Optionen für flexible Anpassungsformen zur Verfügung stehen. Die Frage ist, ob sich durch den veränderten regulatorischen
Handlungsrahmen auch die Muster, mit denen Betriebe auf Nachfrageeinbrüche reagieren, gewandelt haben.

Welche Bedeutung die Instrumente intern- und extern-numerischer Flexibilität für die Anpassung des Arbeitseinsatzes haben und wie sich deren Einsatzmuster geändert haben, soll der nachfolgende Vergleich der Zyklen 1973/79 und 2008/10 zeigen. Der Beitrag rekapituliert zunächst, welche deregulierenden Schritte den Flexibilitätsspielraum am Arbeitsmarkt ausgeweitet haben (Abschnitt 2). Anschließend wird untersucht, wie der Arbeitsmarkt im Abschwung der beiden Konjunkturzyklen reagierte, wie, mit welchen Instrumenten und in welchem Umfang Beschäftigung stabilisiert werden konnte (Abschnitt 3). Abschnitt 4 setzt sich mit einigen Argumenten auseinander, die den Einsatz intern-numerischer Flexibilität kritisch bewerten. Ein kurzes Fazit (Abschnitt 5) rundet den Beitrag ab.

\section{Erweiterter Spielraum für betriebliche Flexibilität}

Gegenüber früheren Konjunktureinbrüchen haben sich die institutionell-rechtlichen Rahmenbedingungen am Arbeitsmarkt erheblich verändert. Der Arbeitsmarkt ist flexibler geworden. Gesetzliche und tarifliche Änderungen dehnten in den Jahren vor dem Konjunkturabschwung 2008/09 Schritt für Schritt den Spielraum für die Betriebe aus, den
Arbeitseinsatz flexibel an eine rückläufige Nachfrage anpassen zu können (Seifert 2006). Die Änderungen betreffen das gesamte Spektrum extern- und intern-numerischer Formen der Flexibilität. Während sich die erstgenannten Formen auf die Variation der Beschäftigtenzahl durch Entlassungen, den Einsatz von Leiharbeit oder befristeten Beschäftigungsverhältnissen beziehen, umfassen Letztere Instrumente der Arbeitszeitverkürzung (Überstundenabbau, Kurzarbeit, Arbeits-

\footnotetext{
1 Im angelsächsischen Raum wird, auch in Abgrenzung zur Weltwirtschaftskrise 1929, die als "Great Depression“ bezeichnet wird, für die aktuelle globale Wirtschaftskrise zunehmend der Begriff "Great Recession“ verwendet. Rampell (2009) ist dem Ursprung dieser Bezeichnung nachgegangen und konnte keinen Urheber ausfindig machen. Interessanterweise fand sie aber heraus, dass alle vorhergehenden wirtschaftlichen Rezessionen irgendwann als große Rezession bezeichnet wurden. Inzwischen wird der Begriff der Großen Rezession auch in Deutschland verwendet, siehe beispielsweise Pieper (2009). Diesen Begriff übernehmen wir, weil es sich in Deutschland um die schwerste Wirtschaftskrise der Nachkriegszeit handelt.
} 
zeitkonten, tarifliche Korridorregelungen). Insgesamt boten sie den 2008 vom abrupten Nachfrageeinbruch mehr oder minder überraschten Betrieben günstigere Voraussetzungen als in früheren Abschwungphasen, zumindest die Stammbelegschaften zu halten. Und umgekehrt erweiterten sie die Anpassungsmöglichkeiten, um bei der Mitte 2009 einsetzenden konjunkturellen Wiederbelebung von Produktion und Dienstleistungen den Arbeitseinsatz rasch und vergleichsweise kostengünstig auszuweiten.

Vor allem folgende Deregulierungsschritte erhöhten die extern-numerische Flexibilität:

- Der Kündigungsschutz wurde gelockert (heraufgesetzter Schwellenwert der Anwendbarkeit);

- bei der Leiharbeit entfielen die Begrenzung der Überlassungsdauer, die zum Zeitpunkt des Abschwungs 1973/75 bei drei Monaten lag, sowie das Synchronisationsund das Wiedereinstellungsverbot;

- die sachgrundlose Befristung, die während des Abschwungs 1973/75 überhaupt nicht zulässig war, wurde von 18 Monaten auf zwei, für neugegründete Betriebe auf vier Jahre ausgeweitet und kann durch Tarifverträge darüber hinaus verlängert werden.

Für vermehrte intern-numerische Flexibilität sorgten vor allem tarifvertragliche Regelungen. Sie erlauben, die Arbeitszeit variabel zu gestalten und so den Arbeitseinsatz kostensenkend und produktivitätssteigernd mit einer volatilen Nachfrage abzustimmen. In erster Linie zählen hierzu Arbeitszeitkonten, die mittlerweile von etwa der Hälfte aller Beschäftigten genutzt werden (Groß/Schwarz 2010).

Hinzu kommen tarifliche Regelungen, die bestehende Regelarbeitszeit zu variieren. In zahlreichen Tarifbereichen verfügen die Betriebsparteien über Optionen, entweder die tarifliche Arbeitszeit innerhalb vorgegebener Grenzen abzusenken oder sie im Rahmen von KorridorRegelungen je nach wirtschaftlicher Lage zu verkürzen und umgekehrt auch zu verlängern (Bispinck/WSI-Tarifarchiv 2009).

An Attraktivität eingebüßt hat demgegenüber das Kurzarbeitergeld. Es ist im Vergleich zum Konjunkturabschwung 1973/75 für Betriebe kostenträchtiger geworden, da sie nun zumindest für die ersten sechs Monate die Sozialversiche- rungsbeiträge (Arbeitgeber- und Beschäftigtenanteil) für die verkürzte Arbeitszeit zu leisten haben, falls nicht Weiterbildungsmaßnahmen durchgeführt werden. In beiden Abschwungphasen wurde die Bezugszeit vorübergehend auf zwei Jahre verlängert (vgl. hierzu auch Bogedan in diesem Heft).

Intern- und extern-numerische Instrumente können sich sowohl ersetzen als auch ergänzen. Insofern erweitern die veränderten rechtlich-institutionellen Regelungen nicht nur den Flexibilitätsspielraum. Sie erhöhen zugleich die Handlungsoptionen, um je nach Problemkonstellation den Instrumenteneinsatz zu differenzieren.

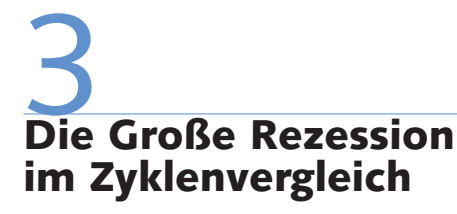

Inwieweit ist es angemessen, bei der Großen Rezession im Vergleich zu früheren Krisenphasen von einer besonderen Entwicklung des Arbeitsmarktes zu sprechen? Inwieweit hat sich das Muster, mit dem der Arbeitsmarkt auf den Nachfrageeinbruch reagierte, geändert? Um Antworten auf diese Fragen zu finden, wird als Nächstes der Abschwung 2008/09 mit dem von 1973/75 verglichen, da beide Konjunktureinbrüche im Hinblick auf ihr Ausmaß am ehesten vergleichbar erscheinen (HerzogStein 2010). Zuvor sind die Wirtschaftszyklen zu definieren, einschließlich ihrer Ab- und Aufschwungphasen. ${ }^{2}$

\subsection{BESTIMMUNG DER KONJUNKTURZYKLEN}

Um das Ausmaß der Großen Rezession in Deutschland erfassen zu können, ist es sinnvoll, sie im Kontext der wirtschaftlichen Entwicklung der letzten 40 Jahre zu spiegeln. ${ }^{3}$ Mit dem Instrument der relativen Output-Lücken - der prozentualen Abweichung des Bruttoinlandsprodukts (BIP) von seinem langfristigen Trend oder Produktionspotenzial - steht ein einfaches und etabliertes Konzept zur Verfügung. ${ }^{4}$

Analog zur Definition eines Aufschwungs (SVR 2007, S. 325f.) wird der Startzeitpunkt eines Abschwungs als jenes Quartal definiert, in dem der Wert der Output-Lücken einen Höhepunkt erreicht hat, in dessen Folge sich die Output-Lücke schließt und vier Quartale lang negativ bleibt. Nach dieser Definition ergeben sich, angelehnt an die Vorgehensweise des Sachverständigenrates zur Begutachtung der gesamtwirtschaftlichen Entwicklung (SVR) (SVR 2007, 2008), seit 1972 sieben Abschwungphasen (Herzog-Stein 2010). Fünf dieser Abschwünge werden als gravierende konjunkturelle Abschwungphasen im Sinne des Sachverständigenrates identifiziert: 2. Quartal 1973 bis 2. Quartal 1975, 4. Quartal 1979 bis 4. Quartal 1982, 1. Quartal 1991 bis 3. Quartal 1993, 1. Quartal 2001 bis 2. Quartal 2005 und die aktuelle Abschwungphase. Deren noch vorläufige Datierung auf Grundlage des derzeitigen Datenmaterials ergibt eine fünf Quartale dauernde Abschwungphase vom 1. Quartal 2008 bis zum 2. Quartal 2009. Seitdem befindet sich die bundesdeutsche Volkswirtschaft in einer wirtschaftlichen Erholungsphase (Abbildung 1, linke Abbildung). ${ }^{5}$

Die relative Outputlücke schrumpfte vom 2008q1 bis 2009q2 um fast sieben Prozentpunkte. Nur im ersten Abschwung 1973/75 hatte sich die Outputlücke ähnlich drastisch verändert und ging innerhalb von acht Quartalen um 5,3 Prozentpunkte zurück. Zudem kam es 1975 mit einer jährlichen Veränderungsrate von - $0,9 \%$ zu dem - mit Ausnahme der aktuellen Abschwungphase - stärksten Rückgang des BIP (Räth 2009, S. 204). In allen anderen Abschwungphasen war der wirtschaftliche Einbruch weniger dramatisch, aber von längerer Dauer. Die Große Rezession war deutlich kürzer als die früheren Abschwungphasen, aber sehr heftig, wie der nachfolgende Abschnitt verdeutlicht.

Da sich im 2. Quartal 2010 die deutsche Volkswirtschaft bereits wieder in einer Erholungsphase befindet, erscheint es angebracht, in den nachfolgenden Abschnitten

2 Alle nachfolgenden quantitativen Analysen basieren auf Daten bis einschließlich 2. Quartal 2010.

3 Der Zeitraum der letzten 40 Jahre, das heißt seit 1970, bietet sich an, da für diesen Zeitraum Zeitreihen auf Quartalsbasis für zahlreiche wichtige ökonomische Kenngrößen, insbesondere auch für das Bruttoinlandsprodukt (BIP), im Rahmen der Volkswirtschaftlichen Gesamtrechnung des Statistischen Bundesamtes zur Verfügung stehen

4 Siehe beispielsweise SVR (2007, 2008); HerzogStein (2010) sowie Sturn/van Treeck in diesem Heft. Für Details bei der Bestimmung der relativen Output-Lücken siehe Herzog-Stein (2010).

5 Diese Datierung ist noch vorläufiger Natur, da zum jetzigen Zeitpunkt noch nicht endgültig feststeht, ob das 2. Quartal 2009 tatsächlich das Ende der aktuellen Abschwungphase markiert. 


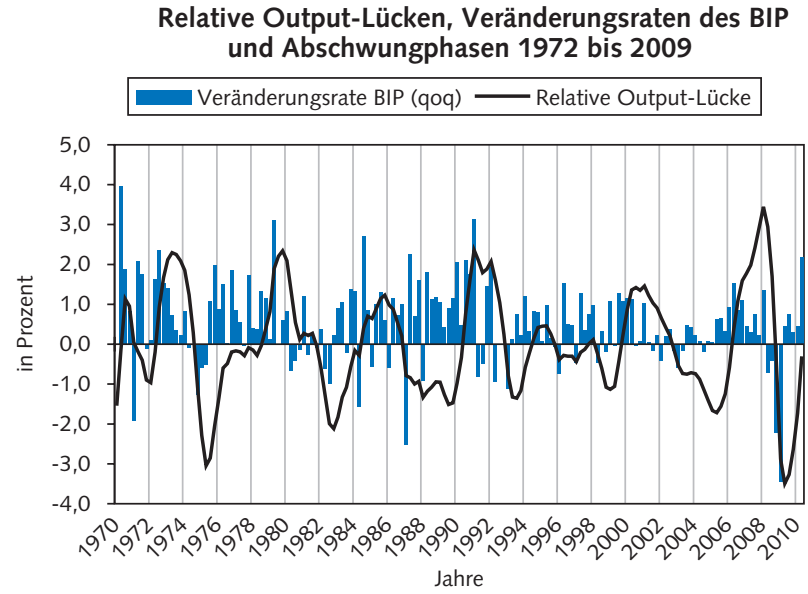

Jahre

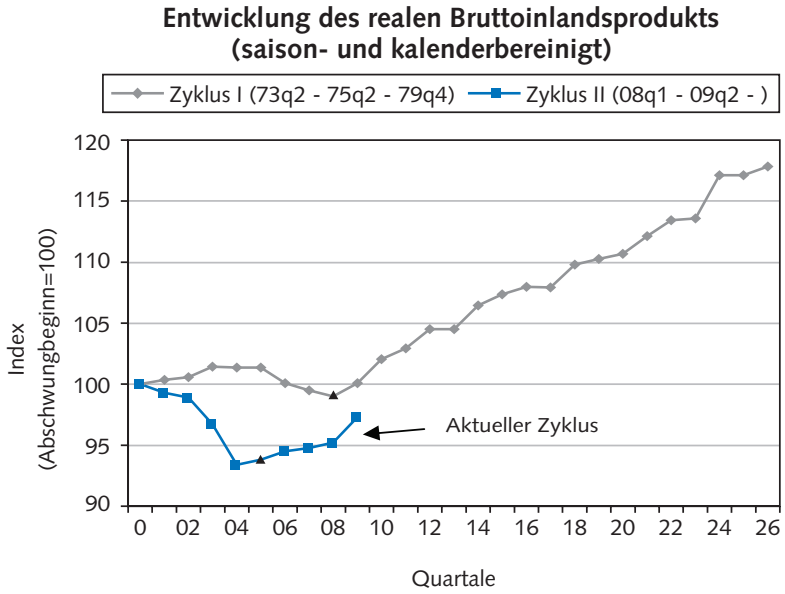

WSI MitTELUNGEN nicht nur die Entwicklung in den beiden Abschwungphasen miteinander zu vergleichen, auch wenn hier der Schwerpunkt der nachfolgenden Analyse liegt - nicht zuletzt aufgrund der noch kurzen Dauer der bisherigen Erholungsphase. Definiert man hierzu die Dauer eines Konjunkturzyklus als die Zeitspanne zwischen zwei konjunkturellen Hochpunkten mit genau einem konjunkturellen Tiefpunkt dazwischen, dann dauerte der erste Konjunkturzyklus vom 2. Quartal 1973 bis zum 4. Quartal 1979, mit dem konjunkturellen Tiefpunkt im 2. Quartal 1975. Der aktuelle begann im 1. Quartal 2008 und dauert noch an, wobei der konjunkturelle Tiefpunkt im 2. Quartal 2009 lag.

\subsection{BRUTTOINLANDSPRODUKT}

In keiner Phase der Nachkriegsentwicklung ist das BIP so stark zurückgegangen wie 2008/09 (um 6,2 \%) (Abbildung 1, rechte Abbildung). Dagegen schrumpfte das BIP 1973/75 „nur“ um 1,0 \%. Allerdings ist zu berücksichtigen, dass im ersten Konjunkturzyklus 1973/79 die Trendwachstumsrate $^{6}$ wesentlich höher war als im gegenwärtigen und deshalb der Vergleich der Entwicklung des BIP das Ausmaß des damaligen wirtschaftlichen Abschwungs nur unzureichend wiedergibt. Legt man die Trendwachstumsrate vor Ausbruch der Krise zugrunde, so belief sich der wirtschaftliche Einbruch in der Großen Rezession auf mehr als $8 \%$ und während des Abschwungs 1973/75 sogar auf mehr als $9 \%$ (vgl. dazu Tabelle 2 im Abschnitt 3.5).
Unterschiede zeigen sich auch in den Aufschwungphasen. Nachdem der aktuelle Konjunkturzyklus nach fünf Quartalen, d.h. im 2. Quartal 2009 seinen Tiefpunkt durchschritt, erholt sich seitdem die Wirtschaft. Bis zum 9. Quartal ist das BIP wieder um 3,7 \% gewachsen, liegt aber noch 2,7 \% unterhalb der Wirtschaftsleistung vom 1. Quartal 2008, dem Beginn des Abschwungs.

Im ersten Zyklus wurde der konjunkturelle Tiefpunkt erst später nach acht Quartalen erreicht, der danach einsetzende Aufschwung dauerte 18 Quartale, und am Ende des Konjunkturzyklus lag das BIP knapp $18 \%$ über dem Niveau vom 2. Quartal 1973, dem Beginn des damaligen Konjunkturzyklus. Deutlich erkennbar ist die damals höhere Dynamik im Vergleich zur aktuellen Entwicklung (Abbildung 1, rechte Abbildung).

\subsection{ARBEITSVOLUMEN}

An der Entwicklung des Arbeitsvolumens lässt sich ablesen, in welchem Ausmaß sich der Arbeitseinsatz änderte. Im aktuellen Abschwung ging das Arbeitsvolumen der Erwerbstätigen innerhalb von fünf Quartalen um 3,2 \% zurück (Abbildung 2, linke obere Abbildung), das der Arbeitnehmer sogar um 3,6\%. Wesentlich stärker fiel der Rückgang in der Abschwungphase 1973/75 aus. Das Arbeitsvolumen schrumpfte innerhalb von fünf Quartalen um 3,3\% (Arbeitnehmer: $-2,9 \%$ ), in der gesamten Abschwungphase, die mit acht Quartalen länger dauerte als aktuell, sogar um 7,6 \% (Arbeitnehmer: $-7,5 \%$ ).
Auch im anschließenden Aufschwung zeigt der Vergleich einen unterschiedlichen Verlauf, soweit die Entwicklung überschaut werden kann. Innerhalb von vier Quartalen expandierte das Arbeitsvolumen um rund $2 \%$, während es im ersten Konjunkturzyklus zunächst auf einem niedrigen Niveau verharrte und selbst zu dessen Ende noch um rund $6 \%$ unter dem Ausgangsniveau zu Krisenbeginn lag.

Da das Arbeitsvolumen das Produkt aus Arbeitszeit je Erwerbstätigen und Erwerbstätigenzahl ist, kann eine ähnliche Entwicklung des Arbeitsvolumens in der jeweiligen Vergleichsperiode das Resultat völlig unterschiedlicher Entwicklungen der beiden Komponenten sein. Nachfolgend wird deshalb die Entwicklung der Arbeitszeit und der Erwerbstätigen in den beiden Konjunkturzyklen genauer betrachtet. Welches Muster zeigt der Zyklenvergleich für die Komponenten des Arbeitsvolumens?

\subsection{ARBEITSZEIT}

Im Abschwung 2008/09 sanken die geleisteten Arbeitsstunden je Erwerbstätigen um 3,4\% (Abbildung 2, linke untere Abbildung), die der Arbeitnehmer sogar um 4,0\%. Etwas stärker mit 4,4 \% je Erwerbstätigen (je Arbeitnehmer: -5,0 \%) fiel der Rückgang der Arbeitszeit in der Abschwungphase des ersten Zyklus aus. Diese Entwicklung zog sich länger hin als im aktuellen Ab-

6 Die Trendrate bezeichnet die langfristige, um konjunkturelle Ausschläge bereinigte wirtschaftliche Entwicklung. 

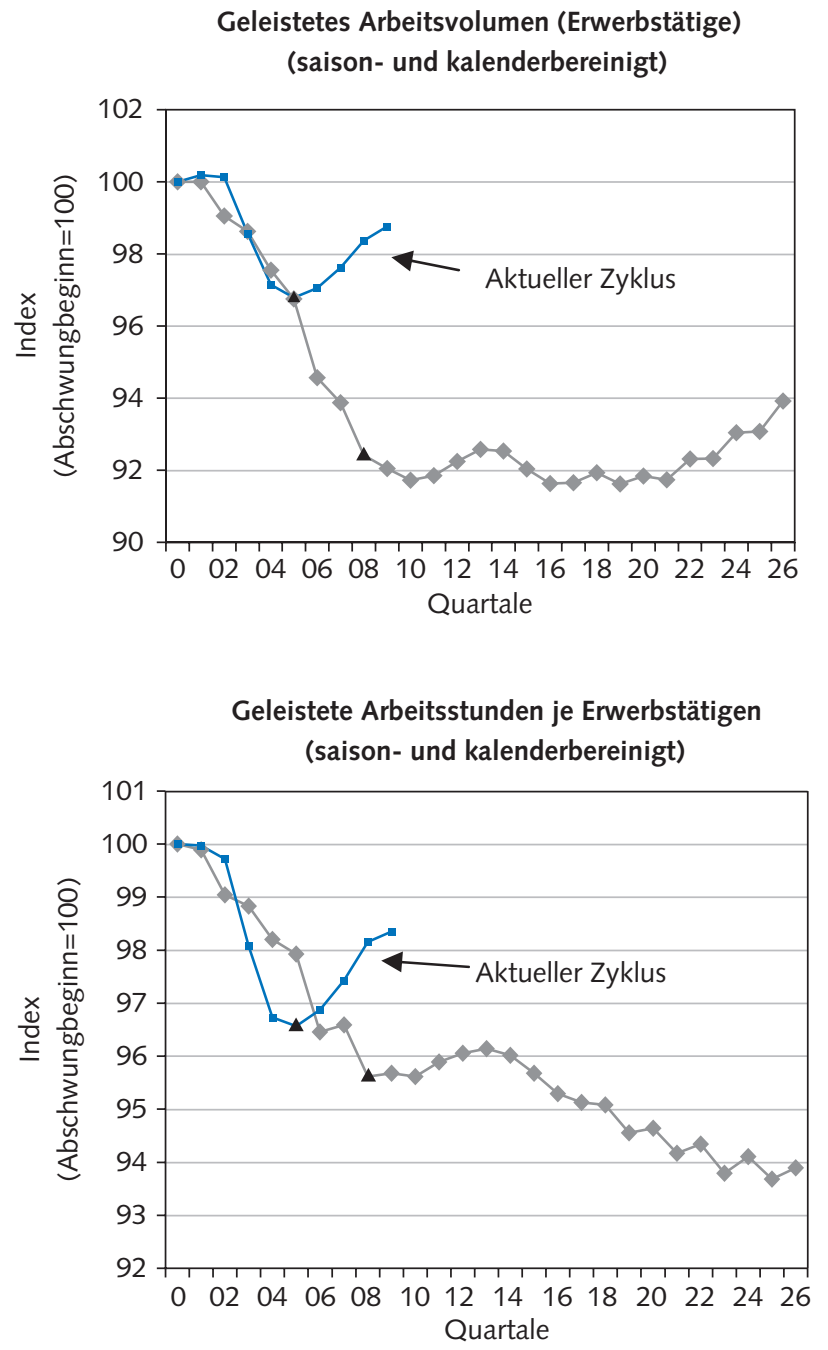

Entwicklung der Erwerbstätigkeit (saison- und kalenderbereinigt)
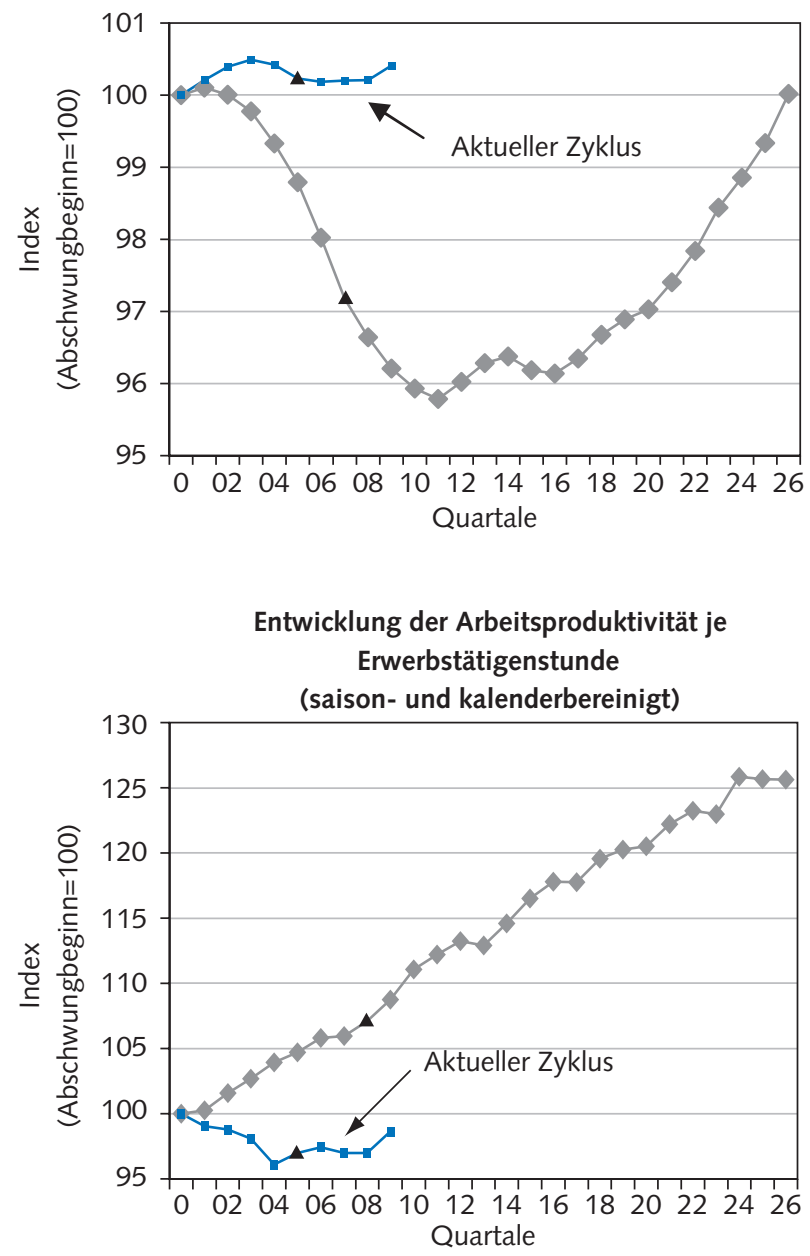

\begin{tabular}{l}
$\longrightarrow$ Zyklus I (73q2 - 75q2 - 79q4) \\
$\longrightarrow$ Zyklus II (08q1 - 09q2 - ) \\
\hline
\end{tabular}

schwung. Eine wesentliche Besonderheit ist vor allem darin zu sehen, dass, anders als im Abschwung 1973/75, die Arbeitszeitreduktion aktuell für den gesamten Rückgang des Arbeitsvolumens verantwortlich ist. Ein weiterer Unterschied in der Anpassung der Arbeitszeit besteht darin, dass im gegenwärtigen Konjunkturzyklus die Zahl der geleisteten Arbeitsstunden pro Kopf nicht nur schneller als im Vergleichszyklus sank, in der wirtschaftlichen Erholung legte sie auch schneller wieder zu. Seit dem Tiefpunkt stieg sie in vier Quartalen um $1,9 \%$ und lag im 2. Quartal 2010 nur noch 1,6\% (je Arbeitnehmer:-1,9\%) unter dem Ausgangsniveau vor Krisenbeginn. Völlig anders verlief die Entwicklung im ersten Konjunkturzyklus, als die geleisteten Ar- beitsstunden je Erwerbstätigen auch während des nachfolgenden Aufschwungs weiter zurückgingen und an dessen Ende 6,1 \% (je Arbeitnehmer:-6,3 \%) unter ihrem Vorkrisenniveau lagen.

Da im Abschwung 1973/75 das Repertoire der zeitlichen Anpassungsmöglichkeiten wesentlich beschränkter war als aktuell (vgl. Abschnitt 2), die geleistete Arbeitszeit pro Kopf aber in vergleichbarem Umfang, wenngleich mit unterschiedlichem Tempo, zurückging, stellt sich die Frage, welche Maßnahmen intern-numerischer Flexibilität den Arbeitseinsatz jeweils reduzierten. Eine Antwort verspricht die Betrachtung der Arbeitszeitkomponenten in den Zeiträumen von 1973 bis 1975 und 2007 bis 2009, die näherungsweise den beiden Abschwungphasen entsprechen (Tabelle 1). ${ }^{7}$

In beiden Abschwungphasen ging die Jahresarbeitszeit je Arbeitnehmer deutlich zurück, im aktuellen Abschwung um 3,3 $\%$ und im Referenzzeitraum 1973 bis 1975 um 4,6\%, das heißt um 44,6 Stunden (Std.) beziehungsweise sogar fast 83 Std. (Tabelle 1, erste Zeile). Diese Unterschiede haben damit zu tun, dass im ersten Zeitraum die Trendkomponente, basierend auf Verkürzungen der tariflichen Wochenar-

7 Zwar liegen die detaillierten Arbeitszeitinformationen der IAB-Arbeitszeitrechnung auch auf Quartalsbasis vor, allerdings sind sie nicht saisonbereinigt. Deshalb wird hier ein Vergleich auf Jahresbasis vorgenommen. 
Tabelle 2: Komponenten der Beschäftigungssicherung in den Abschwungphasen

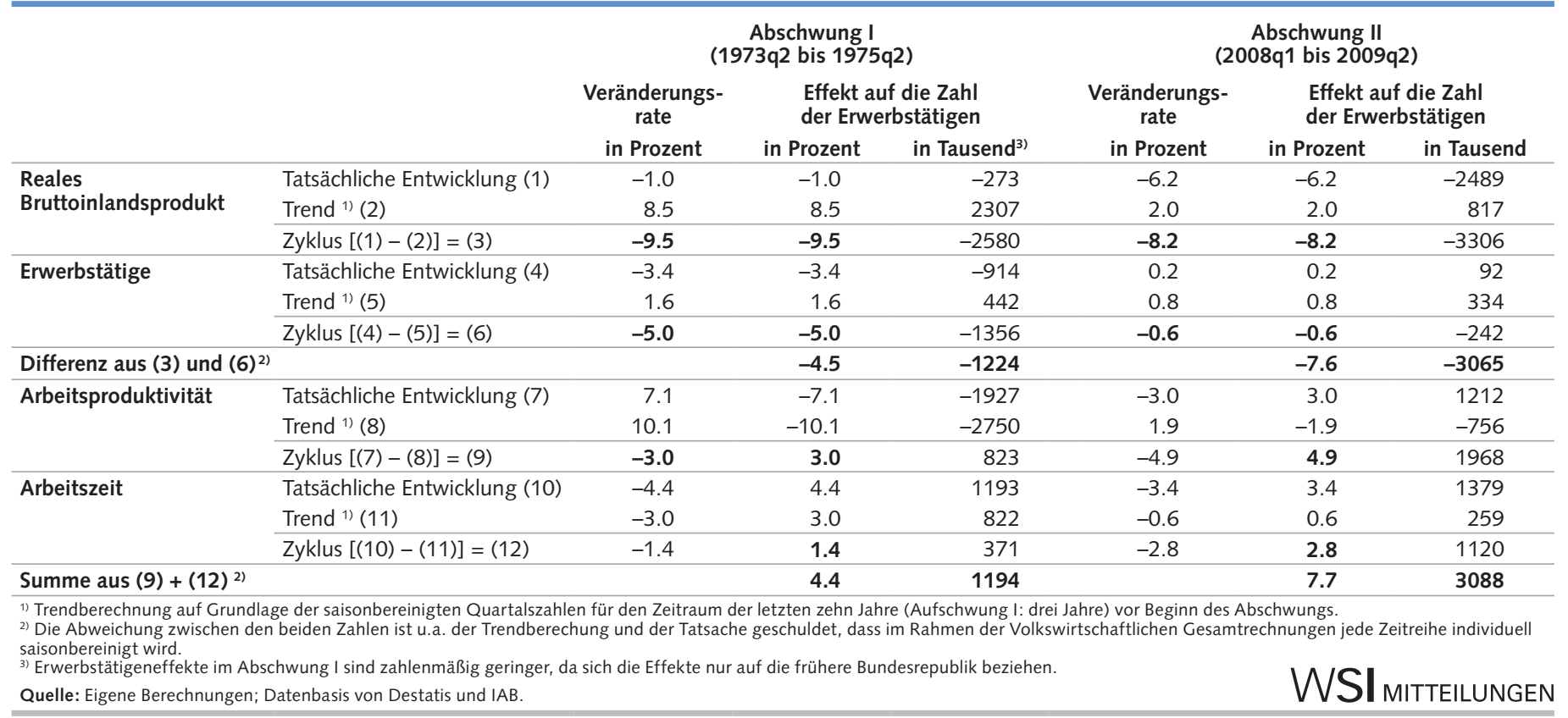

Betrachtet man die zyklischen Veränderungen der BIP-Komponenten, dann lässt sich deren Beitrag zur Beschäftigungssicherung darstellen (Tabelle 2).

Tabelle 2 verdeutlicht, dass im ersten Abschwung die Erwerbstätigenzahl tatsächlich um 3,4\% oder 914.000 Personen abgenommen hat. Berücksichtigt man die trendmäßige Entwicklung der Erwerbstätigen vor Ausbruch des Abschwungs, die mit einem Plus von 1,6\% oder 442.000 rechnerisch veranschlagt wird, ergibt sich insgesamt ein negativer zyklischer Beschäftigungseffekt von fast 1,4 Mio. Personen oder $5 \%$ der Erwerbstätigen. Dieser Wert wäre rein rechnerisch noch erheblich höher auf einen Rückgang von 9,5\% oder fast 2,6 Mio. anzusetzen, wenn nicht erstens die Arbeitszeit zur Stabilisierung der Beschäftigung zusätzlich über den Trend hinaus verkürzt worden wäre. Dieser Schritt sicherte rechnerisch 1,4\% oder 370.000 Arbeitsplätze. Ein weiterer Beitrag zur Beschäftigungssicherung geht auf das Konto der Arbeitsproduktivität, die sich durch das Horten von Arbeit gegenüber ihrem Trend verlangsamte und weitere 3,0\% oder mehr als 800.000 Arbeitsplätze sicherte. Insgesamt wurden somit rund 1,2 Mio. Arbeitsplätze im damaligen Wirtschaftsabschwung gesichert.

Im aktuellen Abschwung nahm dagegen die Zahl der Erwerbstätigen sogar leicht um $0,2 \% \mathrm{zu},{ }^{10}$ obwohl rein rechnerisch aufgrund des Rückgangs des BIP sogar ein zyklischer Beschäftigungseinbruch in der Größenordnung von 8,2 \% aller Erwerbstätigen oder 3,3 Mio. Arbeitsplätzen zu erwarten gewesen wäre.

Dass aber die Erwerbstätigenzahl in den betrachteten sechs Quartalen nicht ab-, sondern noch zugenommen hat, ist auf die beobachtete Reaktion der Arbeitszeit und der Stundenproduktivität zurückzuführen. Die Arbeitszeitverkürzung sicherte - bei Berücksichtung des nach wie vor leicht negativen Arbeitszeittrends - die Beschäftigung von 2,8\% oder etwas über 1,1 Mio. und das Horten von Arbeit sogar von fast $5 \%$ oder knapp 2 Mio. der Erwerbstätigen. Aktuell nahm die Arbeitsproduktivität je Erwerbstätigenstunde bis zum konjunkturellen Tiefpunkt im 2. Quartal 2009 um 3,0 \% ab, und nach acht Quartalen verharrt sie trotz einsetzender Erholung noch immer in etwa auf diesem Niveau (Abbildung 2, rechte untere Abbildung). Ohne Arbeitszeitverkürzungen und das Horten von Arbeit wäre rein rechnerisch die Zahl der Erwerbstätigen während der Großen Rezession um knapp $8 \%$ oder mehr als 3 Mio. gesunken.

Instrumente der intern-numerischen Flexibilität leisteten in den Abschwungphasen beider Konjunkturzyklen einen bedeutenden Beitrag zur Beschäftigungssicherung. In der aktuellen Krise fällt er mit $2,8 \%$ gegenüber $1,4 \%$ doppelt so hoch aus, obwohl die Arbeitszeit in einem etwas geringeren Umfang gesunken ist. Der höhere Beschäftigungseffekt erklärt sich dadurch, dass im Abschwung 1973/75 ein größerer relativer Anteil der Arbeitszeitreduktion auf die Trendkomponente entfällt. ${ }^{11}$ In den Jahren vor der aktuellen Krise hatte sich der Arbeitszeittrend stark verlangsamt. Demgegenüber leisteten 2008/09 die darüber hinausgehenden, aus rein konjunkturellen Gründen erfolgten Anpassungen der Arbeitszeit einen wesentlich größeren Beitrag zur Beschäftigungssicherung. Dieser Effekt hat mit der gewandelten Komposition der verwendeten Instrumente zu tun. Tarifliche Vereinbarungen hatten das Repertoire der zeitlichen Anpassungsmöglichkeiten erweitert. Quantitativ bedeutsam war ferner in beiden Abschwüngen das Horten von Arbeitskräften.

\section{SEKTORALE BETRACHTUNG}

Das hier gezeichnete Bild einer ausschließlich auf interner Flexibilität und Horten von Arbeitskräften vollzogenen Bewältigung der Krise trügt allerdings insofern, da es ausschließlich die globale Perspektive reflektiert. Unterschiedliche oder gar gegenläufige sektorale Entwicklungen der Beschäftigung werden in diesem Blickwinkel nivelliert. So weist beispielsweise das besonders stark von den externen Schocks getroffene Produzierende Gewerbe, dessen reale Wertschöpfung im ersten Abschwung um gut $5 \%$ und im zweiten sogar um 21,6\%

10 Der Arbeitsmarkt reagiert stets mit Verzögerungen auf Änderungen in der Produktion.

11 Vergleiche hierzu Fußnote 8. 
absackte, in beiden Abschwungphasen Beschäftigungsverluste auf. Die Zahl der Erwerbstätigen ist zwischen dem 1. Quartal 2008 und dem 2. Quartal 2009 um knapp 2,2 \% gesunken und liegt trotz wirtschaftlicher Belebung im 2. Quartal 2010 rund 5,0\% unter dem Niveau zu Krisenbeginn.

Doch auch diese Daten unterzeichnen das tatsächliche Ausmaß des rückläufigen Arbeitseinsatzes in diesem Bereich. Nicht erfasst bleiben die hier eingesetzten Leiharbeitnehmer, die den Dienstleistungen zugerechnet werden. Es ist sicherlich keine unrealistische Annahme, den überwiegenden Anteil der insgesamt zwischen dem 1. Quartal 2008 und dem 2. Quartal 2009 saisonbereinigt um über $22 \%$ geschrumpften Anzahl an Leiharbeitnehmern dem verminderten Arbeitskräftebedarf im Produzierenden Gewerbe zuzuschreiben. ${ }^{12}$ Insofern geht ein nicht unbeträchtlicher Teil des reduzierten Arbeitseinsatzes auch auf Maßnahmen der extern-numerischen Flexibilität zurück. ${ }^{13}$ Da gleichzeitig die Erwerbstätigkeit in dem quantitativ bedeutsameren Bereich der öffentlichen und privaten Dienstleister durchgängig in den Jahren 2008 bis zur Mitte 2010 weiter expandierte, konnte diese Entwicklung den Rückgang im Produzierenden Gewerbe mehr als kompensieren. ${ }^{14}$

Im Referenzabschwung 1973/75 fiel der Beschäftigungsrückgang im Produzierenden Gewerbe mit 7,5\% stärker aus und wurde auch nicht durch Beschäftigungszuwächse in den Dienstleistungsbereichen kompensiert. Leiharbeit war 1973, kurz nach Inkrafttreten des Arbeitnehmerüberlassungsgesetzes im August 1972, mit rund 30.000 Leiharbeitnehmern noch unbedeutend. Gleichwohl zeigt der von einem niedrigen Niveau ausgehende starke Rückgang um etwa zwei Drittel, dass diese Beschäftigungsform von Beginn an als Konjunkturpuffer diente.

Obwohl der Handlungsspielraum für extern-numerische Flexibilität zwischen den beiden Abschwungphasen gewachsen ist, haben die Betriebe in der aktuellen Krise weniger als in der Vergangenheit auf diese Anpassungsstrategie gesetzt. Zwar hat die Bedeutung von Leiharbeit als Instrument extern-numerischer Anpassung zugenommen, aber der Abbau von Stammarbeitskräften scheint, soweit die Datenlage Aussagen zulässt, weniger zur Anpassung des Arbeitseinsatzes beige- tragen zu haben. Dies kann damit zu tun haben, dass die neuen und erweiterten Instrumente intern-numerischer Flexibilität für die Betriebe kostengünstigere Alternativen bieten und deshalb als funktionale Äquivalente zu extern-numerischer Anpassung dienen. Für diese Annahme spricht, dass 2010 - laut der aktuellen WSIBetriebsrätebefragung - im Investitionsund Gebrauchsgütergewerbe, das vom Nachfragerückgang besonders betroffenen ist, $33 \%$ der Betriebe (mit Betriebsrat), auf die $60 \%$ aller dort Beschäftigten entfallen, Vereinbarungen über Beschäftigungssicherung gelten, die für einen bestimmten Zeitraum betriebsbedingte Kündigungen ausschließen. Im Tausch gegen vermehrte Flexibilität bei der Arbeitszeit und beim Entgelt verzichten Betriebe auf Optionen der Personalanpassung bei den Stammarbeitskräften (Seifert/MassaWirth 2005).

\subsection{HORTEN VON ARBEITSKRÄFTEN}

Das Horten von Arbeitskräften wird vor allem auf die Sorge der Unternehmen zurückgeführt, bei einem neuerlichen Aufschwung einen Mangel an Fachkräften zu erleiden, was angesichts der demografischen Entwicklung keineswegs abwegig ist (Möller 2010; OECD 2010). Die demografischen Vorzeichen haben sich gegenüber der Perspektive im Konjunkturaufschwung nach 1975 komplett umgedreht. Während die Betriebe seinerzeit ein noch wachsendes Erwerbspersonenpotenzial im Hintergrund wussten, stehen sie aktuell einem schrumpfenden gegenüber. Deswegen dürfte sich das Interesse der Unternehmen verstärkt haben, firmenspezifisches $\mathrm{Hu}-$ mankapital möglichst zu sichern.

Das Argument vom Horten von Arbeitskräften ist allerdings zu relativieren. Die Angaben über die Produktivitätsentwicklung basieren auf Berechnungen über die Arbeitszeit, die sich neben statistisch erhobener Daten aus verschiedenen Quellen auch auf Schätzungen stützen (Koch 2001). Die daraus resultierenden Unsicherheiten haben zum einen mit der gewachsenen Bedeutung veränderter Arbeitszeitformen (Zeitkonten, tarifliche Korridorregelungen usw.) zu tun, deren Saldenänderungen gerade in Krisenphasen nur unzureichend erfasst sind. Für die Gesamtwirtschaft ist beispielsweise unbekannt, in welchem Umfang Beschäftigte in der letzten Aufschwungphase Zeitguthaben angelegt und in welchem Umfang sie diese Guthaben im Abschwung aufgelöst und Zeitschulden gebildet haben. Ferner fehlen Daten über die Ausnutzung tariflicher Regelungen zur Verkürzung der Arbeitszeit. Kaum abzuschätzen ist außerdem, in welchem Ausmaß Betriebe ihre in der Boomphase im Rahmen tariflicher Korridorregelungen verlängerten Arbeitszeiten wieder zurückgenommen haben. ${ }^{15}$ Trendextrapolationen liefern unsichere Ergebnisse, wenn es zu Verhaltensänderungen bei der Arbeitszeitgestaltung kommt. Das Fehlen einer regelmäßigen, detaillierten Arbeitszeiterhebung macht sich schmerzlich bemerkbar. Angesichts dieser statistischen Lücken und Schätzunsicherheiten ist nicht auszuschließen, dass aktuell das tatsächliche Ausmaß der Arbeitszeitverkürzungen unterschätzt wird. Je größer jedoch der Schätzfehler, desto geringer ist der auf das Horten von Arbeitskräften zurückgehende Effekt zu veranschlagen und desto geringer fällt auch der Rückgang der Stundenproduktivität aus.

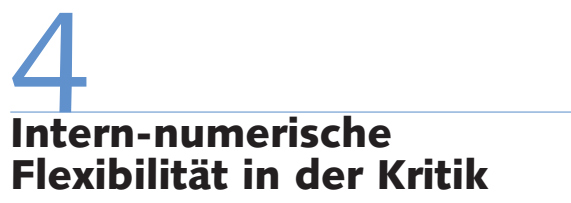

Trotz des beachtlichen beschäftigungspolitischen Erfolges sind Maßnahmen der intern-numerischen Flexibilität nicht

12 Vermutlich dürfte der Einsatz von Leiharbeitnehmern im Produzierenden Gewerbe noch drastischer ausgefallen sein, als es die Gesamtentwicklung indiziert, da sie den Saldo gegenläufiger Entwicklungen in den Wirtschaftsbereichen darstellt. Die amtlichen Daten erfassen nicht den branchenmäßigen Einsatz der Leiharbeitnehmer, der sich deshalb nur mit Abstrichen abschätzen lässt. Hinweise liefert das IAB-Betriebspanel. Danach sank die Zahl der Leiharbeitnehmer im Produktionssektor zwischen dem 30.6.2008 und dem 30.6.2009 um $29 \%$, während sie in den sozialen Dienstleistungen um $34 \%$ stieg (Hohendanner 2010).

13 Aufgrund der unzureichenden Datenlage lässt sich die Entwicklung befristeter Beschäftigungsverhältnisse in den beiden Zyklen nicht vergleichen.

14 Eine detaillierte Analyse der Beschäftigtenentwicklung in den verschiedenen Wirtschaftsbranchen liefert der Beitrag von Rosemann/Kirchmann in diesem Heft.

15 So hatte 2007, als noch nicht der Höhepunkt des Konjunkturaufschwungs erreicht war, ein knappes Viertel der Betriebe mit Betriebsrat die Arbeitszeit im Rahmen tariflicher Korridor-Regelungen ausgeweitet und konnte dann im nachfolgenden Abschwung die Arbeitszeit von einem erhöhten Niveau aus verkürzen (Seifert 2010). 
ohne kritische Einwände geblieben. So wird argumentiert, die Auflösung von Zeitguthaben auf Arbeitszeitkonten würde bei den Betrieben Kosten verursachen, weil sie trotz verkürzter Arbeitszeiten den auf die Regelarbeitszeit bezogenen Lohn zu bezahlen hätten (Walwei 2010). Sieht man einmal von den einmaligen Kosten für die Einführung und den laufenden für die Pflege von Zeitkonten ab, dann ist dieses Instrument im Hinblick auf die Lohnkosten kostenneutral oder sogar kostensenkend, da es als Anpassungsinstrument häufig zuschlagpflichtige Überstundenarbeit ersetzt. Die monatlichen Einkommen entsprechen der über einen vereinbarten längeren Zeitraum durchschnittlich geleisteten Arbeitszeit. Bei der Bildung von Guthaben profitieren die Arbeitgeber, da die Arbeitnehmer durch nicht ausgezahlte Einkommensbestandteile, die unverzinst bleiben, in Vorleistung treten. Generell erlauben Zeitkonten einen effizienteren Einsatz von Arbeit, der sich rasch und kostengünstig mit der Nachfrage synchronisieren lässt. Leerzeiten lassen sich ebenso wie Lagerkosten reduzieren (Seifert 2005).

Gegen Kurzarbeit und damit im Prinzip auch gegen alle übrigen Formen Beschäftigung sichernder Arbeitszeitverkürzung wird eingewendet, dass hierdurch Fehlanreize gesetzt würden, die die gegebene Beschäftigungsstruktur konservieren und den Strukturwandel hemmen würden (Eichhorst/Marx 2009; OECD 2010). Dieser Effekt könnte dann eintreten, wenn Arbeitskräfte ohne Aussicht auf Rückkehr zur Normalarbeitszeit gehalten würden und gleichzeitig in anderen Bereichen offene Stellen nicht besetzt werden könnten. Diese
Konstellation ist unwahrscheinlich. Kürzere Arbeitszeiten sind mit Einkommenseinbußen verbunden und signalisieren außerdem unsichere Beschäftigungsperspektiven. Deshalb besteht für Beschäftigte ein doppelter Anreiz, auf Arbeitsplätze mit Normalarbeitszeit zu wechseln. Solange aber eine schwache Arbeitskräftenachfrage keine attraktiven Beschäftigungsalternativen bietet, stellen Beschäftigung sichernde Arbeitszeitverkürzungen allemal die bessere Alternative gegenüber der ansonsten drohenden Arbeitslosigkeit dar. Der weiterhin bestehende Bezug zur Arbeitswelt schützt vor Verlust von beruflichen und sozialen Qualifikationen und sichert die Beschäftigungsfähigkeit. Sofern absehbar ist, dass Arbeitsplätze selbst nach konjunktureller Erholung wegfallen werden, können kürzere Arbeitszeiten in Verbindung mit auf den externen Arbeitsmarkt ausgerichteter Qualifizierung nahtlose Übergänge verbessern und Mismatch-Arbeitslosigkeit vermeiden helfen.

Schließlich ist zu sehen, dass Beschäftigung sichernde Arbeitszeitanpassungen in Form von Überstundenabbau, Zeitkonten oder tariflichen Reduktionen nicht nur eine Reihe von Kostenvorteilen für Betriebe bieten (Herzog-Stein/Seifert 2010). Sie entlasten auch die öffentlichen Haushalte, vor allem die Arbeitslosenversicherung, die weder Kurzarbeiter- noch Arbeitslosengeld zu zahlen hat. Die antizyklische Steuerung von Zeitkonten stabilisiert außerdem die Einkommen und damit den privaten Konsum.

\section{Fazit}

Der Zyklenvergleich hat gezeigt, dass Instrumente intern-numerischer Flexibilität Tradition haben, in konjunkturellen Abschwungphasen den Arbeitseinsatz an eine rückläufige Nachfrage anzupassen. Gewandelt hat sich die Bedeutung der Instrumente. In der aktuellen Krise geht der Rückgang des gesamtwirtschaftlichen Arbeitsvolumens allein auf eine entsprechend gesunkene Arbeitszeit zurück. Den größten Beitrag leisten in beiden Abschwüngen Änderungen der tariflichen/betriebsüblichen Arbeitszeit. Unterschiede zeigt der Instrumentenmix. Während Kurzarbeit in den beiden hier verglichenen Zyklen eine ähnlich bedeutsame Rolle für die Beschäftigungssicherung spielte, hat der Abbau von Überstunden an Bedeutung verloren; die Betriebe favorisieren stattdessen Arbeitszeitkonten. Überraschend ist, dass die erweiterten Möglichkeiten externnumerischer Flexibilität offensichtlich nur relativ mäßig genutzt wurden, obwohl der regulatorische Rahmen mehr Spielraum bietet als in den 1970er Jahren. Dagegen hat das Horten von Arbeitskräften an Bedeutung gewonnen. Allerdings basieren diese Berechnungen auf unsicheren Daten, die auf dringenden Forschungsbedarf verweisen. Die zunehmende Flexibilisierung der Arbeitszeit erfordert eine gesicherte Datenbasis, wie sie eine regelmäßige und differenzierte Arbeitszeiterhebung liefern könnte. Aufhellung verlangen auch die betrieblichen Motive und Kalküle für das Horten von Arbeitskräften. 
Bach, H.-U./Kohler, H./Reyher, L./Teriet, B. (1977): Arbeitszeit und Arbeitsvolumen in der Bundesrepublik Deutschland 1960-1975, in: Mitteilungen aus der Arbeitsmarkt- und Berufsforschung (MittAB) 1, S. 19-37

Bispinck, R./WSI-Tarifarchiv (2009): Tarifliche Regelungen zur befristeten Arbeitszeitverkürzung, Elemente qualitativer Tarifpolitik 67, Düsseldorf

Eichhorst, W./Marx, P. (2009): Kurzarbeit: Sinnvoller Konjunkturpuffer oder verlängertes Arbeitslosengeld?, IZA Standpunkte 5, Bonn Groß, H./Schwarz, M. (2010): Arbeitszeit, Altersstrukturen und Corporate Social Responsibility, Wiesbaden

Herzog-Stein, A. (2010): Das Jahr der Krise, in: Groß, H./Seifert, H. (Hrsg.): Zeitkonflikte, Berlin, S. 17-36

Herzog-Stein, A./Seifert H. (2010): Stabile Beschäftigung durch flexible Arbeitszeiten, in: Arbeit - Zeitschrift für Arbeitsforschung, Arbeitsgestaltung und Arbeitspolitik (im Erscheinen)

Hohendanner, C. (2010): Unsichere Zeiten, unsichere Verträge?, IAB-Kurzbericht 14, Nürnberg

Koch, S. (2001): Arbeitszeitkonten stabilisieren die Beschäftigung, IAB-Kurzbericht 4, Nürnberg

Krugman, P. (2009): Free to Lose, in: New York Times vom 13.

November

Möller, J. (2010): The German labor market response in the world recession - de-mystifying a miracle, in: Zeitschrift für ArbeitsmarktForschung 4, S. 325-336

Organisation für wirtschaftliche Zusammenarbeit und Entwicklung (OECD) (2010): Employment Outlook, Paris
Piecha, M. (1975): Die Tarifbewegungen im Jahre 1974, in: WSI-Mitteilungen 3, S. 129-138

Pieper, N. (2009): Die Große Rezession - Amerika und die Zukunft der Weltwirtschaft, München

Rampell, C. (2009): "Great Recession“: A Brief Etymology, in: New York Times vom 11. März

Räth, N. (2009): Rezessionen in historischer Betrachtung, in: Statistisches Bundesamt (Hrsg.): Wirtschaft und Statistik 3, S.203-208 Sachverständigenrat zur Begutachtung der gesamtwirtschaftlichen Entwicklung (SVR) (2007): Das Erreichte nicht verspielen, Jahresgutachten 2007/2008, Ziffern 481-492, Wiesbaden

Sachverständigenrat zur Begutachtung der gesamtwirtschaftlichen Entwicklung (SVR) (2008): Die Finanzkrise meistern - Wachstumskräfte stärken, Jahresgutachten 2008/2009, Ziffern 475 - 490, Wiesbaden Seifert, H. (2005): Arbeitszeitpolitischer Modellwechsel: von der Normalarbeitszeit zu kontrollierter Flexibilität, in: Ders. (Hrsg.): Flexible Zeiten in der Arbeitswelt, Frankfurt/New York, S. 40-66

Seifert, H. (2006): Was hat die Flexibilisierung des Arbeitsmarktes gebracht?, in: WSI-Mitteilungen 11, S. 601-608

Seifert, H. (2010): Beschäftigungssichernde Arbeitszeitverkürzungen. Intermezzo oder arbeitszeitpolitischer Aufbruch? in: Ders. (Hrsg.): Zeitkonflikte, Berlin, S. 37-52

Seifert, H./Massa-Wirth, H. (2005): Pacts for employment and competitiveness in Germany, in: Industrial Relations Journal 3, S. 217-240 Walwei, U. (2010): Folgen der Finanzkrise: Wie robust ist der Arbeitsmarkt?, in: Arbeit - Zeitschrift für Arbeitsforschung, Arbeitsgestaltung und Arbeitspolitik (im Erscheinen) 